\title{
Langevin Approach to Fractional Diffusion Equations including Inertial Effects
}

\author{
R. Friedrich, S. Eule*, F. Jenko \\ September 17, 2018 \\ Institute of Theoretical Physics \\ Westfälische Wilhelms Universität \\ Münster \\ Wilhelm-Klemm-Str. 9 \\ G-48149 Münster
}

\begin{abstract}
In recent years, several fractional generalizations of the usual KramersFokker-Planck equation have been presented. Using an idea of Fogedby [H.C. Fogedby, Phys. Rev. E 50, 041103 (1994), we show how these equations are related to Langevin equations via the procedure of subordination.
\end{abstract}

Introduction. - Some 70 years ago, Kramers 1 considered the motion of a Brownian particle subject to a space-dependent force $\mathbf{F}(\mathbf{x})$ per unit mass. His goal was to compute the joint probability distribution $f(\mathbf{x}, \mathbf{u}, t)$ for finding a particle at time $t$ at the position $\mathbf{x}$ with the velocity $\mathbf{u}$. For this quantity he could derive the famous Kramers-Fokker-Planck (KFP) equation [2, 3]

$$
\left[\frac{\partial}{\partial t}+\mathbf{u} \cdot \nabla_{x}+\mathbf{F}(\mathbf{x}) \cdot \nabla_{u}\right] f(\mathbf{x}, \mathbf{u}, t)=\mathcal{L}_{\mathrm{FP}} f(\mathbf{x}, \mathbf{u}, t)
$$

where $\mathcal{L}_{\mathrm{FP}}$ is the Fokker-Planck collision operator

$$
\mathcal{L}_{\mathrm{FP}} f=\gamma \nabla_{u} \cdot(\mathbf{u} f)+D \Delta_{u} f .
$$

As is well known, Eq. (11) corresponds to the Langevin equations (see, e.g., Ref. [2]

$$
\frac{d}{d t} \mathbf{x}(t)=\mathbf{u}(t), \quad \frac{d}{d t} \mathbf{u}(t)=\mathbf{F}(\mathbf{x})-\gamma \mathbf{u}(t)+\boldsymbol{\Gamma}(\mathrm{t})
$$

where $\boldsymbol{\Gamma}(\mathrm{t})$ obeys white noise statistics. It describes a Brownian particle which is subject to the relation $\left\langle\mathbf{x}^{2}\right\rangle \sim D t$ where $D$ is the diffusion coefficient. In many complex systems, this relation is violated, however. In fact, one often finds $\left\langle\mathbf{x}^{2}\right\rangle \sim D_{\alpha} t^{\alpha}$ with $\alpha \neq 1$ which is described as "anomalous" or "strange" diffusion. Here, $D_{\alpha}$ is a generalized diffusion coefficient with units $\left[D_{\alpha}\right]=m^{2} s^{-\alpha}$. Depending on $\alpha$, such a process is called subdiffusive $(\alpha<1)$, superdiffusive $(1<\alpha<2)$, ballistic $(\alpha=2)$, or turbulent-diffusive $(\alpha=3)$.

\footnotetext{
*yeahhart@uni-muenster.de, Tel.:+49-251-8334924, Fax: +49-251-8336328
} 
As was shown by several authors, strange diffusion may be described by fractional generalizations of Eq. (1) (for a review including discussions of various applications see e.g., Ref. 4]). However, the latter may differ in the way the fractional character is incorporated. Thus it comes as no surprise that three different types of fractional KFP equations may be found in the literature. E.g., Metzler and Klafter [5, 6, 7], proposed the equation

$$
\frac{\partial f(\mathbf{x}, \mathbf{u}, t)}{\partial t}=\left[-\mathbf{u} \cdot \nabla_{x}-\mathbf{F}(\mathbf{x}) \cdot \nabla_{u}+\mathcal{L}_{\mathrm{FP}}\right] \gamma_{\delta} D_{t}^{1-\delta} f(\mathbf{x}, \mathbf{u}, t)
$$

which they obtained by means of a non-Markovian generalization of the ChapmanKolmogorov equation. Another kind of fractional KFP equation has been proposed by Barkai and Silbey [8, namely

$$
\left[\frac{\partial}{\partial t}+\mathbf{u} \cdot \nabla_{x}+\mathbf{F}(\mathbf{x}) \cdot \nabla_{u}\right] f(\mathbf{x}, \mathbf{u}, t)=\mathcal{L}_{\mathrm{FP}} \gamma_{\delta} D_{t}^{1-\delta} f(\mathbf{x}, \mathbf{u}, t)
$$

where $\gamma_{\delta}$ is a damping coefficient whose units are $\left[\gamma_{\delta}\right]=s^{\delta-1}$ and $D_{t}^{1-\delta}$ is the fractional time derivative whose Laplace space representation reads $D_{t}^{1-\delta} \leftrightarrow$ $\lambda^{1-\delta}$. Finally, employing the concept of continuous time random walks (CTRWs), Friedrich and co-workers [9, 10, were able to derive the equation

$$
\left[\frac{\partial}{\partial t}+\mathbf{u} \cdot \nabla_{x}+\mathbf{F}(\mathbf{x}) \cdot \nabla_{u}\right] f(\mathbf{x}, \mathbf{u}, t)=\mathcal{L}_{\mathrm{FP}} \gamma_{\delta} \mathcal{D}_{t}^{1-\delta} f(\mathbf{x}, \mathbf{u}, t) .
$$

Here, $\mathcal{D}_{t}^{1-\delta}$ denotes a fractional substantial derivative which can be written in Laplace space as $\mathcal{D}_{t}^{1-\delta} \leftrightarrow\left[\lambda+\mathbf{u} \cdot \nabla_{x}+\mathbf{F}(\mathbf{x}) \cdot \nabla_{u}\right]^{1-\delta}$.

In this letter we address the important question of how these three fractional KFP equations are connected to each other. As is well known, fractional diffusion equations can be linked to CTRWs. [11] And according to Fogedby [12, the latter can in turn be linked to sets of Langevin equations. This fact will be exploited below in order to gain insight into the nature of the stochastic processes underlying the three scenarios that were just described. For the sake of simplicity, we restrict ourselves to one-dimensional problems with no external force.

Langevin approach to fractional diffusion equations. - In the spirit of Fogedby, let us first consider a stochastic process which is described by the following system of Langevin equations:

$$
\frac{d}{d s} u(s)=-\gamma u(s)+\Gamma(s), \quad \frac{d}{d s} t(s)=\eta(s) .
$$

Here, the variable $s$ is to be interpreted as an internal time, whereas $t$ is the physical (wall-clock) time. Moreover, $\Gamma$ and $\eta$ are described, respectively, by Gaussian and one-sided Lévy stable distributions [denoted by $L_{\delta}(x)$ ] [13. Mathematically speaking, the stochastic process $u(s)$ is subordinated by the $t(s)$ process. The latter is invertible, and the probability density of finding the internal time $s$ at time $t$ is given by

$$
p(s, t) \propto \frac{d}{d s}\left[1-L_{\delta}\left(t /\left(\gamma_{\delta} s\right)^{1 / \delta}\right)\right]
$$

which is called the inverse one-sided Lévy stable distribution. It is a solution of the equation

$$
\frac{\partial}{\partial t} p(s, t)=-\frac{\partial}{\partial s} \gamma_{\delta} D_{t}^{1-\delta} p(s, t),
$$


and its Laplace transform reads $\hat{p}(s, \lambda) \propto \lambda^{\delta-1} \exp \left(-\gamma_{\delta} \lambda^{\delta} s\right)$. Assuming that the stochastic processes $t(s)$ and $u(s)$ are statistically independent, the probability $P(u, t)$ of finding the velocity $u$ at time $t$ can be written as

$$
P(u, t)=\int_{0}^{\infty} P_{0}(u, s) p(s, t) d s
$$

where the distribution function $P_{0}(u, s)$ is a solution of the standard diffusion equation,

$$
\frac{\partial P_{0}(u, s)}{\partial s}=\mathcal{L}_{\mathrm{FP}} P_{0}(u, s) .
$$

From Eqs. (9)-(11) it then follows that $P(u, t)$ satisfies the fractional diffusion equation

$$
\frac{\partial P(u, t)}{\partial t}=\mathcal{L}_{\mathrm{FP}} \gamma_{\delta} D_{t}^{1-\delta} P(u, t) .
$$

The idea of representing the solution of a fractional diffusion equation like Eq. (12) as a superposition of Gaussians goes back to Barkai [14. In the following, we will extend this method from velocity space to phase (position-velocity) space.

The fractional KFP equation by Metzler and Klafter. - Let us now consider the Langevin system

$$
\frac{d}{d s} x(s)=u(s), \quad \frac{d}{d s} u(s)=-\gamma u(s)+\Gamma(s), \quad \frac{d}{d s} t(s)=\eta(s)
$$

which is closely related to Eq. (3). Here, both $x(s)$ and $u(s)$ are subordinated by the same $t(s)$ process. In analogy with Eq. (10), the probability distribution $f(x, u, t)$ can be written as

$$
f(x, u, t)=\int_{0}^{\infty} f_{0}(x, u, s) p(s, t) d s
$$

where $f_{0}(x, u, s)$ is the solution of the Fokker-Planck equation

$$
\left[\frac{\partial}{\partial s}+u \frac{\partial}{\partial x}\right] f_{0}(x, u, s)=\mathcal{L}_{\mathrm{FP}} f_{0}(x, u, s) .
$$

Using these relations together with Eq. (91), one obtains

$$
\frac{\partial f(x, u, t)}{\partial t}=\left[-u \frac{\partial}{\partial x}+\mathcal{L}_{\mathrm{FP}}\right] \gamma_{\delta} D_{t}^{1-\delta} f(x, u, t) .
$$

This is the fractional generalization of the usual KFP equation considered by Metzler and Klafter [5, 6, 7. Thus we have shown that the corresponding stochastic process is given by Eq. (13).

The fractional KFP equation by Barkai and Silbey. - Next, we want to consider the Langevin system

$$
\frac{d}{d t} x(t)=u(t), \quad \frac{d}{d s} u(s)=-\gamma u(s)+\Gamma(s), \quad \frac{d}{d s} t(s)=\eta(s) .
$$


Here, the velocity coordinate is subordinated by the internal time process while the evolution of the space variable is in physical time. These equations may be rewritten as

$$
\frac{d}{d s} x(s)=u(s) \eta(s), \quad \frac{d}{d s} u(s)=-\gamma u(s)+\Gamma(s), \quad \frac{d}{d s} t(s)=\eta(s) .
$$

For any specific realization of $\eta(s)$, one can view this as a stochastic process which only depends on the Gaussian variable $\Gamma(s)$. The corresponding probability distribution $f_{0}(x, u, s)$ is subject to the KFP-type equation

$$
\left[\frac{\partial}{\partial s}+u \eta(s) \frac{\partial}{\partial x}\right] f_{0}(x, u, s)=\mathcal{L}_{\mathrm{FP}} f_{0}(x, u, s) .
$$

The solution of this equation is a Gaussian probability distribution with the second order moments defined by

$$
\begin{aligned}
\frac{d}{d s}\left\langle u^{2}\right\rangle(s) & =-2 \gamma\left\langle u^{2}\right\rangle(s)+2 D, \\
\frac{d}{d s}\langle x u\rangle(s) & =-\gamma\langle x u\rangle(s)+\eta(s)\left\langle u^{2}\right\rangle(s), \\
\frac{d}{d s}\left\langle x^{2}\right\rangle(s) & =2 \eta(s)\langle x u\rangle(s) .
\end{aligned}
$$

For simplicity, we first consider the case $\gamma=0$ in which one obtains

$$
\begin{aligned}
\left\langle u^{2}\right\rangle(s(t)) & =2 D s(t), \\
\frac{d}{d t}\langle x u\rangle(s(t)) & =\left\langle u^{2}\right\rangle(s(t)), \\
\frac{d}{d t}\left\langle x^{2}\right\rangle(s(t)) & =2\langle x u\rangle(s(t)) .
\end{aligned}
$$

Introducing the auxiliary variables $\sigma(t)$ and $\Sigma(t)$ via

$$
\frac{d}{d t} \sigma(t)=s(t), \quad \frac{d}{d t} \Sigma(t)=\sigma(t),
$$

one finds

$$
\left\langle u^{2}\right\rangle(s(t))=2 D s(t), \quad\langle x u\rangle(s(t))=2 D \sigma(t), \quad\left\langle x^{2}\right\rangle(s(t))=4 D \Sigma(t),
$$

and the characteristic function

$$
Z(k, \alpha, \cdot)=\int d x \int d u f(x, u, \cdot) \exp [i k x+i \alpha u]
$$

of $f_{0}$ is obtained as

$$
Z_{0}(k, \alpha, s, \sigma, \Sigma)=\exp \left[-D \alpha^{2} s(t)-2 D \alpha k \sigma(t)-2 D k^{2} \Sigma(t)\right] .
$$

[We note that the latter can also be calculated directly from Eq. (17).]

Now, the stochastic process $\eta(s)$ defines a probability distribution $W(s, \sigma, \Sigma, t)$ where $s(t), \sigma(t)$, and $\Sigma(t)$ are related to $\eta(s)$ via Eqs. (18) and (22). We assume that this function satisfies the equation

$$
\left[\frac{\partial}{\partial t}+\sigma \frac{\partial}{\partial \Sigma}+s \frac{\partial}{\partial \sigma}\right] W(s, \sigma, \Sigma, t)=-\frac{\partial}{\partial s} \gamma_{\delta} D_{t}^{1-\delta} W(s, \sigma, \Sigma, t)
$$


which is the natural generalization of Eq. (9). In analogy with Eq. (10), the generic characteristic function of $f(x, u, t)$ can thus be written as

$$
Z(k, \alpha, t)=\int d s \int d \sigma \int d \Sigma Z_{0}(k, \alpha, s, \sigma, \Sigma) W(s, \sigma, \Sigma, t) .
$$

It is straightforward to show that the corresponding distribution function $f(x, u, t)$ obeys the fractional KFP equation

$$
\left[\frac{\partial}{\partial t}+u \frac{\partial}{\partial x}\right] f(x, u, t)=\mathcal{L}_{\mathrm{FP}} \gamma_{\delta} D_{t}^{1-\delta} f(x, u, t)
$$

with $\gamma=0$.

The case $\gamma \neq 0$ is only slightly more difficult. Eq. (28) yields

$$
\left[\frac{\partial}{\partial t}-k \frac{\partial}{\partial \alpha}\right] Z(k, \alpha, t)=-\left[\gamma \alpha \frac{\partial}{\partial \alpha}+D \alpha^{2}\right] \gamma_{\delta} D_{t}^{1-\delta} Z(k, \alpha, t) .
$$

Using Eq. (25), we note that

$$
\left[k \frac{\partial}{\partial \alpha}\right] Z_{0}=\left[\sigma \frac{\partial}{\partial \Sigma}+s \frac{\partial}{\partial \sigma}\right] Z_{0}
$$

and

$$
\left[\gamma \alpha \frac{\partial}{\partial \alpha}+D \alpha^{2}\right] Z_{0}=\left[(2 \gamma s-1) \frac{\partial}{\partial s}+\gamma \sigma \frac{\partial}{\partial \sigma}\right] Z_{0} .
$$

By means of Eq. (27), we then obtain

$$
\left[\frac{\partial}{\partial t}+\sigma \frac{\partial}{\partial \Sigma}+s \frac{\partial}{\partial \sigma}\right] W(s, \sigma, \Sigma, t)=\left[\frac{\partial}{\partial s}(2 \gamma s-1)+\gamma \frac{\partial}{\partial \sigma} \sigma\right] \gamma_{\delta} D_{t}^{1-\delta} W(s, \sigma, \Sigma, t)
$$

which is a generalization of Eq. (9). So, starting with Eq. (17) and assuming that the stochastic process $\eta(s)$ defines a probability distribution $W(s, \sigma, \Sigma, t)$ which satisfies Eq. (32), the fractional KFP equation à la Barkai and Silbey [Eq. (28)] holds.

The fractional KFP equation by Friedrich et al. - Finally we address the generalized KFP equation with retardation proposed in Ref. [9]. It reads

$$
\left[\frac{\partial}{\partial t}+u \frac{\partial}{\partial x}\right] f(x, u, t)=\mathcal{L}_{\mathrm{FP}} \gamma_{\delta} \mathcal{D}_{t}^{1-\delta} f(x, u, t)
$$

where $\mathcal{D}_{t}^{1-\delta}$ is the fractional substantial derivative introduced above. According to Ref. [10], this equation can also be written as

$$
\left[\frac{\partial}{\partial t}+u \frac{\partial}{\partial x}\right] f(x, u, t)=\mathcal{L}_{\mathrm{FP}} \int_{0}^{t} Q\left(t-t^{\prime}\right) e^{-\left(t-t^{\prime}\right) u \partial x} f\left(x, u, t^{\prime}\right) d t^{\prime}
$$

if the memory kernel $Q\left(t-t^{\prime}\right)$ is chosen appropiately. In this case, the characteristic function satisfies the equation

$$
\begin{aligned}
& {\left[\frac{\partial}{\partial t}-k \frac{\partial}{\partial \alpha}\right] Z(k, \alpha, t)=} \\
& -\int_{0}^{t} Q\left(t-t^{\prime}\right)\left[\gamma \alpha \frac{\partial}{\partial \alpha}+D \alpha^{2}\right] Z\left(k, \alpha+k\left(t-t^{\prime}\right), t^{\prime}\right) d t^{\prime},
\end{aligned}
$$


and one finds

$$
Z_{0}\left(k, \alpha+k\left(t-t^{\prime}\right), s, \sigma, \Sigma\right)=\exp \left[-D \alpha^{2} s-2 D \alpha k \tilde{\sigma}-2 D k^{2} \tilde{\Sigma}\right]
$$

where we have introduced the new variables $\tilde{\sigma}=\sigma+s\left(t-t^{\prime}\right)$ and $\tilde{\Sigma}=\Sigma+\sigma(t-$ $\left.t^{\prime}\right)+s\left(t-t^{\prime}\right)^{2} / 2$. Using relations similar to those in Eqs. (30) and (31) as well as the ansatz (27), we obtain the evolution equation

$$
\begin{aligned}
{\left[\frac{\partial}{\partial t}\right.} & \left.+\sigma \frac{\partial}{\partial \Sigma}+s \frac{\partial}{\partial \sigma}\right] W(s, \sigma, \Sigma, t) \\
& =\int_{0}^{t} Q\left(t-t^{\prime}\right)\left[\frac{\partial}{\partial s}(2 \gamma s-1)+\gamma \frac{\partial}{\partial \sigma} \sigma\right] \times \\
& \times W\left(s, \sigma-s\left(t-t^{\prime}\right), \Sigma-\sigma\left(t-t^{\prime}\right)+\frac{s}{2}\left(t-t^{\prime}\right)^{2}, t^{\prime}\right) d t^{\prime}
\end{aligned}
$$

for $W(s, \sigma, \Sigma, t)$. The latter is simply a retarded version of Eq. (32). To clarify the difference between Eq. (32) and Eq. (37) we introduce the shifted variables $\hat{\sigma}=\sigma-s t$ and $\hat{\Sigma}=\Sigma-\sigma t+\frac{s}{2} t^{2}$ in the sense that

$$
W(s, \sigma, \Sigma, t)=\tilde{W}\left(s, \sigma-s t, \Sigma-\sigma t+\frac{s}{2} t^{2}, t\right)
$$

holds. Consequently Eq. (37) can be written as

$$
\frac{\partial}{\partial t} \tilde{W}(s, \sigma, \Sigma, t)=\int_{0}^{t} Q\left(t-t^{\prime}\right)\left[\frac{\partial}{\partial s}(2 \gamma s-1)+\gamma \frac{\partial}{\partial \sigma}(\sigma+s t)\right] \tilde{W}\left(s, \sigma, \Sigma, t^{\prime}\right) d t^{\prime} .
$$

The difference in the two approaches of Barkai and Silbey and Friedrich et al. can be traced back to the Langevin process (22). Integration yields for instance

$$
\sigma(t)=\int_{0}^{t} d t^{\prime} s\left(t^{\prime}\right)=\int_{0}^{s(t)} d s^{\prime} s^{\prime} \eta\left(s^{\prime}\right)
$$

where $\eta(s)$ is a stochastic process. Since one has to integrate over the product $s \eta(s)$ a stochastic interpretation of this integral is needed. A similar situation arises in stochastic processes involving multiplicative white noise sources, where different interpretations of similar integrals have been given by Ito and Stratonovich (for a discussion see e.g. 2]). We conjecture that different interpretations of this integral and the integral arising for the variable $\Sigma(t)$ lies at the origin of the two different fractional equations for the probability distributions $W(s, \sigma, \Sigma, t)$.

Conclusions. - Three different types of fractional generalizations of the KFP equation describing anomalous diffusion of inertial particles can be found in the literature. Based on the idea of subordination, which is equivalent to the introduction of an intrinsic, fluctuating time, we have clarified the meaning of these different equations. Whereas in the approach of Metzler and Klafter [5] - 7] both position and velocity depend on the intrinsic time, the approach of Barkai and Silbey [8 and Friedrich et. al. 9], 10 assumes that only the velocity is subjected to the subordination procedure. We conjecture that the difference between the approaches of Barkai and Silbey and Friedrich et al. is due to different interpretations of stochastic integrals. 


\section{References}

[1] H.A. Kramers, Physica 7, 284 (1940)

[2] H. Risken, The Fokker-Planck Equation (Springer, Berlin, 1989)

[3] N.G. van Kampen, Stochastic Processes in Physics and Chemistry (NorthHolland, Amsterdam, 1981)

[4] W. T. Coffey, Y. P. Kalmykov and J. T. Waldron, The Langevin Equation (World Scientific, Singapore, 2004)

[5] R. Metzler and J. Klafter, J. Chem. Phys. B 104, 3851 (2000)

[6] R. Metzler and J. Klafter, Phys. Rev. E 61, 6308 (2000)

[7] R. Metzler, Phys. Rev. E 62, 6233 (2000)

[8] E. Barkai and R. Silbey, J. Chem. Phys. B 104, 3866 (2000)

[9] R. Friedrich, F. Jenko, A. Baule and S. Eule Phys. Rev. Lett. 96, 230601 (2006)

[10] R. Friedrich, F. Jenko, A. Baule, and S. Eule, Phys. Rev. E 74, 041103 (2006)

[11] R. Metzler and J. Klafter, Phys. Rep. 339, 1 (2000)

[12] H.C. Fogedby, Phys. Rev. E 50, 1657 (1994)

[13] W. Feller, An Introduction to Probablity Theory and Its Applications, Vol.2 (Wiley, New York, 1970)

[14] E. Barkai, Phys. Rev. E 63, 046118 (2001) 